\title{
A theory of a heterogeneous divisible commodity exchange economy ${ }^{\text {is }}$
}

\author{
Farhad Husseinov* \\ Department of Economics, Bilkent University, 06800 Bilkent, Ankara, Turkey
}

\section{A R T I C L E I N F O}

\section{Article history:}

Received 12 March 2010

Received in revised form 26 October 2010

Accepted 3 December 2010

Available online 14 December 2010

\section{JEL classification:}

D51

C71

\section{Keywords:}

Heterogeneous divisible commodity

Exchange economy

Land trading economy

Competitive equilibrium

Core

Fair division

\begin{abstract}
A B S T R A C T
In theoretical land economics the existence of a competitive equilibrium with an additive price is considered problematic. This paper studies the exchange and allocation of a heterogeneous divisible commodity such as land, which is modeled as a measurable space. In a 'land' trading economy with unordered convex preferences, the existence of a competitive equilibrium with an additive equilibrium price is proved. This paper demonstrates also the existence of a weak core and a fair allocation.
\end{abstract}

(c) 2010 Elsevier B.V. All rights reserved.

\section{Introduction}

We consider the exchange of a heterogeneous divisible commodity, such as land, which is modeled as a measurable space $(X$, $\Sigma$ ). In theoretical models of land economics, $X$ is assumed to be a Borel measurable subset of the Euclidean space $R^{2}$ (or more generally, $R^{k}$ ) and $\Sigma$ to be the Borel $\sigma$-algebra $\mathcal{B}(X)$ of subsets of $X$. It is usual to consider this measurable space with the Lebesgue measure. The existence of a competitive equilibrium with additive prices in land trading has been an issue in theoretical land economics; this is the central question that the present paper addresses.

The first study of competitive equilibria in the traditions of general equilibrium theory (à la Arrow-Debreu), in a land-trading economy is due to Berliant (1985). He shows the existence of a competitive equilibrium when preferences over land parcels are represented by utility functions of the form $U(B)=\int_{B} u(x) d x$, so that $U$ is a measure on $\mathcal{B}(X)$ absolutely continuous with respect

\footnotetext{
is I am grateful to the participants of the session in the International Conference on Game Theory at Stony Brook, July 10-14, 2006, where results of an early draft of this paper were presented, for useful discussions. I am also grateful to Nedim Alemdar and Ozgur Evren for many useful comments and a referee for many useful suggestions that led to a number of improvments.

* Tel.: +90 312 2902228; fax: +90 3122665140.

E-mail address: farhad@bilkent.edu.tr
}

to the Lebesgue measure. His proof uses a method that imbeds the land-trading economy into an economy with the commodity space $L_{\infty}(X)$, and then uses Bewley's (1972) equilibrium existence results along with methods of infinite dimensional analysis.

Dunz (1991) studies the existence of the core in a land-trading economy for substantially more general preferences. In this paper preferences are represented by the utility functions that are compositions of quasi-concave functions with a finite number of parcel characteristics. Dunz proves that under these assumptions on preferences the weak core of a land-trading game is nonempty. These characteristics are countably additive over land parcels. Assigning a finite number of additive characteristics to land parcels is a common assumption made in empirical literature on land trading. Dunz (1991), based on results of his joint work with Berliant and Dunz's (1986), argues that “...if prices are required to be additive... then an equilibrium might not exist. If no equilibrium with additive prices exists, then it is not clear what the final allocation of the economy will be since there would always be arbitrage opportunities. This suggests that competitive equilibrium might not be the appropriate solution concept for economies with land." However, nonexistence of equilibrium in Berliant and Dunz's (1986) example is of the same nature as one in the classical case of trading divisible commodities and is due to nonconvexity of preferences. One of the goals of the present paper is to show that a competitive equilibrium with an additive price exists in a land-trading economy with rather general unordered ‘convex' preferences. In fact, 
this is done in the more abstract context of a measurable-spacetrading economy. We show the existence of an equilibrium where the equilibrium price is a measure, $v$, on $(X, \Sigma)$, absolutely continuous with respect to the sum of all characteristic measures. For the land-trading economy, where all characteristic measures are assumed to be absolutely continuous with respect to the Lebesgue measure $\lambda$, we obtain that the equilibrium price $v$ is also absolutely continuous with respect to the Lebesgue measure $\lambda$. Hence, the Radon-Nikodim derivative $(d \nu / d \lambda)$ is an integrable function $h$ on the measure space $(X, \Sigma, \lambda)$ (see Aliprantis and Border, 1994, p. 350). So $v(B)=\int_{B} h(x) d \lambda(x)$ for all measurable sets $B$ in $X$. Here the function $h$ can be interpreted as the equilibrium price density on $X$.

In a related paper Berliant and Dunz (2004) (henceforth, BD) study the existence of equilibrium in a model where the shape and location of land parcels affect agents' preferences. While BD assume complete and transitive preferences, we do not employ these assumptions in the present paper. Another difference between the two papers is that preferences in BD are defined directly on the $\sigma$-algebra of land pieces, whereas here they are defined through characteristic measures of land pieces. The use of the characteristic measures allows us to formulate the convexity of agents' preferences in a straightforward way. By contrast, the existence theorem of BD utilizes a novel 'convexity' condition called 'separation by hyperplanes' which is assumed for nonwasteful partitions. Moreover, they assume the existence of nonwasteful partitions. Actually, the last two assumptions are joint assumptions on agents' preferences. Thus, there are significant conceptual and methodological differences between the two papers.

Using the standard scheme, in this paper we also show that, a competitive allocation is a weak core allocation. This core existence result generalizes existing core existence results in two directions: first, it considers the division problem in the setting of an abstract measurable space and does not assume a certain reference measure, and second, preferences are not assumed to be ordered.

The next topic dealt with in this paper is the existence of a fair division. Examples of the fair division problem include dividing an inheritance fairly among the inheritors, and designing land reform laws that allow dividing land owned by a collective farm fairly among its members in transition economies. On a deeper level, fairness can be regarded as an essential and a desirable property of a solution concept in economics (and game theory).

Weller (1985) considers a problem of fair division of a measurable space $(X, \Sigma)$ with a finite number of atomless measures describing agents' preferences over measurable subsets. He shows the existence of an envy-free and efficient partition of this problem. In a somewhat different setting, namely when $X$ is a measurable subset of the Euclidean space $R^{k}$ and preference measures are nonatomic and absolutely continuous with respect to the Lebesgue measure, Berliant et al. (1992) show the existence of a groupenvy-free and efficient partition. The concept of a group-envy-free partition is stronger than the concept of an envy-free partition. However, neither of these results implies the other. Weller's result is concerned with the more abstract problem of fair partitioning an abstract measurable space with no reference measure. On the other hand, Berliant et al. (1992) prove the existence of a fair partition in a stronger sense. Our approach to the fairness problem is abstract and we will consider much more general preferences over measurable pieces. The result established here contains both of the above-discussed results.

We would like to stress that all the results of this paper assume rather general classes of preferences over measurable pieces. The importance of considering such preferences in the present context has been indicated by students of heterogeneous commodity markets. For example, Chambers (2005) notes that "working with more general domains of utility functions should be a motivating goal in this model."

In proving the existence of a competitive equilibrium we use the following scheme: first, we show that the problem of exchange of a heterogeneous divisible commodity is reducible to that of a finite number of homogeneous divisible commodities (totality of subjectively attributed characteristics of measurable pieces), where endowments are subsets in the commodity space rather than commodity bundles. Then we transform this economy into the general model introduced by Gale and Mas-Colell (1975), and thereby employ their competitive equilibrium existence theorem.

This introduction is followed by a section that introduces basic concepts and some preliminary results. In Section 3 we present our central results on the existence of a competitive equilibrium and nonemptiness of the core in a measurable-space-trading economy. Section 4 studies fairness criteria for this economy. In Appendix A we state a classical theorem on convexity of the range of a vectormeasure mapping, defined on partitions of a measurable space that is used in the proofs of the main results. Appendix B is devoted to the proofs of the main results.

\section{Preliminaries}

We model a measurable space trading problem in the following way. Let $(X, \Sigma)$ be a measurable space (a cake or land plot) and let $P=\left\{A_{1}, A_{2}, \ldots, A_{n}\right\}$ be a measurable ordered partition of $X$. Let $\mu_{1}, \mu_{2}, \ldots, \mu_{n}$ be nonatomic finite vector measures on $(X, \Sigma)$ of dimensions $s_{1}, s_{2}, \ldots, s_{n}$, respectively. $\mu_{i}^{j}\left(j=1, \ldots, s_{i}\right)$ will denote the $j$-th component of vector-measure $\mu_{\mathrm{i}}$. The interpretation is that there are $n$ persons, denoted as $1,2, \ldots, n$, each contributing his share $A_{i}(i \in N=\{1,2, \ldots, n\})$ to the cake $X$, and pieces of the cake are valued by individuals according to their measures $\mu_{1}, \mu_{2}, \ldots$, $\mu_{n}$, respectively. The components of vector $\mu_{i}(B)$ are interpreted as measures of different (possibly subjective) attributes of a measurable piece $B$, attached to this piece by individual $i$. We assume that individual $i$ has a preference $\succ_{i}$ over his subjective attributes profiles $\mu_{i}(B), B \in \Sigma$, and hence over measurable sets $B \in \Sigma$. We will use the same symbol $\succ_{i}$ for denoting both of these preferences. No confusion should arise. Every ordered measurable division $\left\{B_{1}, \ldots\right.$, $\left.B_{n}\right\}$ of $X$ will be interpreted as a feasible allocation of $X$. All divisions considered further are assumed to be ordered and measurable. An alternative interpretation is that initially individuals possess land parcels $A_{1}, \ldots, A_{n}$, respectively, and they exchange pieces of land to improve their welfare.

Definition 2.1. A pair $\left(P=\left\{B_{1}, B_{2}, \ldots, B_{n}\right\}, v\right)$ consisting of a division $P$ and a measure $v$ is a competitive equilibrium if for each individual $i$ the subset $B_{i}$ maximizes his preference $\succ_{i}$ on his budget set

$\mathcal{B}_{i}(\nu)=\left\{B \in \Sigma \mid \nu(B) \leq \nu\left(A_{i}\right)\right\}$.

In this case, the division $P$ is called an equilibrium allocation and the measure $v$ is called an equilibrium price.

A coalition is an arbitrary nonempty subset of $N$. The set of all coalitions is denoted as $\mathcal{N}$.

Definition 2.2. A coalition $I \subset N$ improves (weakly improves) upon a division $P=\left\{B_{1}, B_{2}, \ldots, B_{n}\right\}$ if there exists a division $Q=\left\{C_{i} \mid i \in I\right\}$ of $A(I)=\cup_{i \in I} A_{i}$ such that $C_{i} \succ{ }_{i} B_{i}$ for all $i \in I$ (not $B_{i} \succ{ }_{i} C_{i}$ for all $i \in I$ and $C_{i} \succ{ }_{i} B_{i}$ at least for one $i \in I$.)

Definition 2.3. Division $P=\left\{B_{1}, B_{2}, \ldots, B_{n}\right\}$ is a weak core allocation (core allocation) if no coalition improves (weakly improves) upon $P$. The set of all (weak) core allocations is the (weak) core of the measurable space trading economy. 
Next, we introduce two concepts of Pareto efficiency of a division.

Definition 2.4. Division $P=\left\{B_{1}, B_{2}, \ldots, B_{n}\right\}$ of $X$ is weak Pareto efficient (Pareto efficient) if there is no division $P^{\prime}=\left\{B_{1}^{\prime}, B_{2}^{\prime}, \ldots, B_{n}^{\prime}\right\}$ of $X$ such that $\mu_{i}\left(B_{i}^{\prime}\right) \succ_{i} \mu_{i}\left(B_{i}\right)$ for all $i \in N$ (not $B_{i} \succ_{i} B_{i}^{\prime}$ for all $i \in N$ and $B_{i}^{\prime} \succ_{i} B_{i}$ for at least one $\left.i \in N\right)$.

We will identify a vector of vectors (perhaps of different dimensions) as a long vector with scalar coordinates arranged in the lexicographic order. Sometimes we will denote coordinates with double indexes, the first showing the component vector and the second showing the component in that component vector.

\section{Existence of a competitive equilibrium and core}

In this section for preferences $\succ_{i}(i \in N)$ on the nonnegative orthant, $R_{+}^{S_{i}}$, in the Euclidean space $R^{s_{i}}$ we denote $P_{i}\left(x_{i}\right)=$ $\left\{x_{i}^{\prime} \in R_{+}^{S_{i}} \mid X_{i}^{\prime} \succ_{i} X_{i}\right\}$. Clearly, the correspondence $P_{i}$ defines $\succ_{i}$ in a unique way. We do not assume preferences $\succ_{i}$ are complete or transitive. We assume that preferences $\succ_{i}, i \in N$, are continuous (that is, graphs of correspondences $P_{i}$ are open relative to $R_{+}^{S_{i}} \times R_{+}^{S_{i}}$ ) and convex (that is, the upper contour sets, $P_{i}\left(x_{i}\right)$, are convex for arbitrary characteristics vectors $\left.x_{i} \in R_{+}^{s_{i}}, i \in N\right)$.

As usual for vectors $x=\left(x_{1}, \ldots, x_{m}\right), y=\left(y_{1}, \ldots, y_{m}\right)$ in $R^{m}$ we write $x \geq y$ if $x_{i} \geq y_{i}$ for all $i=1, \ldots, m$. We write $x>y$ if $x \geq y$ and $x \neq y$, and $x \gg y$ if $x_{i}>y_{i}$ for all $i=1, \ldots, m$.

We will assume also that preferences are monotonic: if $x_{i}, x_{i}^{\prime} \in R_{+}^{s_{i}}, x_{i}^{\prime} \geq x_{i}$, then $P_{i}\left(x_{i}^{\prime}\right) \subset P_{i}\left(x_{i}\right)$.

We assume the following about the initial endowments of the individuals.

Assumption. (Positive Endowments)

For each $i \in N$, the set $A_{i}$ can be divided into $n$ measurable parts $A_{i j}(j \in N)$ such that $\mu_{j}\left(A_{i j}\right) \gg 0$ for all $j \in N$.

One property, with a clear economic interpretation, that is sufficient for fulfilment of the positive endowments assumption may be formulated in terms of mutual continuity of measures $\mu_{i}(i \in N)$. For two measures $\mu$ and $\mu^{\prime}$ defined on the same domain, $\mu^{\prime}$ is called absolutely continuous with respect to $\mu$ if $\mu(A)=0$ implies $\mu^{\prime}(A)=0$. It is easily seen that if for each $i \in N$ there exists $j \in\left\{1, \ldots, s_{i}\right\}$ such that $\mu_{i}^{j}\left(A_{i}\right)>0$ and all the component measures are absolutely continuous with respect to each other, then the assumption of positive endowments is satisfied.

The central result of this paper is the following competitive equilibrium existence theorem.

Theorem 3.1. If the attribute vector-measures $\mu_{i}, i \in N$, are nonatomic, and preferences $\succ_{i}, i \in N$, are irreflexive, continuous, monotone and convex, and the positive endowments assumption is satisfied, then there exists a competitive equilibrium $\left(P=\left\{B_{1}, B_{2}, \ldots\right.\right.$, $\left.\left.B_{n}\right\}, v\right)$ in the measurable space trading economy. Moreover, the equilibrium price measure $v$ is absolutely continuous with respect to the sum of all component measures of vector-measures $\mu_{i}, i \in N$.

Proof of this theorem will be given in Appendix B.

Corollary 3.2. Under the assumptions of Theorem 3.1 the weak core in the measurable space trading economy is nonempty.

Proof. It is easy to see that a competitive equilibrium allocation $\left\{B_{1}, B_{2}, \ldots, B_{n}\right\}$ belongs to the weak core. Assume on the contrary, that there exists a coalition $I$ that improves upon partition $\left\{B_{1}, B_{2}\right.$, $\left.\ldots, B_{n}\right\}$. Thus there exists a partition $\left\{C_{i} \mid i \in I\right\}$ of $A(I)=\cup_{i \in I} A_{i}$ such that $C_{i} \succ{ }_{i} B_{i}$ for all $i \in I$. Then since $\left(\left\{B_{1}, B_{2}, \ldots, B_{n}\right\}, v\right)$ is an equilibrium we have $v\left(C_{i}\right)>v\left(B_{i}\right)$ for all $i \in I$. Adding these inequalities we will get $v(C(I))>v(A(I))$. This contradicts to $C(I)=A(I)$.
Hüsseinov (2008) shows that under the assumptions of nonatomicity of characteristic measures $\mu_{i}(i \in N)$, and rationality, continuity and convexity of preferences, the weak core of the heterogeneous divisible commodity exchange economy is nonempty. In Corollary 3.2 preferences are not assumed to be rational. Instead, monotonicity of preferences and positive endowments are assumed.

It follows from Theorem 5 in Hüsseinov (2008) that if in addition to the assumptions of Theorem 3.1 preferences are rational and measures $\sum_{j=1}^{s_{i}} \mu_{i}^{j}(i \in N)$ are absolutely continuous with respect to each other, then the weak core and the core coincide. From this observation and Corollary 3.2 it follows that under these assumptions the core is nonempty.

The following proposition is proved in Hüsseinov (2008, see Theorem 5).

Proposition 3.3. If preferences $\succ_{i}$ are the strict parts of rational continuous weak preferences $\succcurlyeq_{i}$, monotone (for $x_{i}, x_{i}^{\prime} \in R_{+}^{s_{i}}, x_{i}^{\prime}>$ $x_{i}$ implies $x_{i}^{\prime} \succ_{i} x_{i}$, ) and if measures $\eta_{i}=\sum_{j=1}^{s_{i}} \mu_{i}^{j}(i \in N)$ are absolutely continuous with respect to each other, then the weak core and the core coincide.

Corollary 3.2 and Proposition 3.3 imply

Corollary 3.4. If in addition to the assumptions of Proposition 3.3 preferences are convex and positive endowments assumption is satisfied, then the core in the measurable space trading economy is nonempty.

\section{Existence of fair divisions}

Definition 4.1. A division $P=\left\{A_{1}, A_{2}, \ldots, A_{n}\right\}$ of $X$ is fair if it is

(a) weakly Pareto efficient, that is, if there is no other division $Q=\left\{C_{1}, C_{2}, \ldots,\left(C_{n}\right)\right\}$ such that $\mu_{i}\left(C_{i}\right) \in P_{i}\left(\mu_{i}\left(A_{i}\right)\right)$ for $i \in N$, and

(b) envy-free, that is, if $\mu_{i}\left(A_{j}\right) \notin P_{i}\left(\mu_{i}\left(A_{i}\right)\right)$ [in other words, not $\left.A_{j} \succ_{i} A_{i}\right]$ for all $i, j \in N$.

We define now a stronger version of the last concept.

Definition 4.2. A division $\left\{A_{1}, A_{2}, \ldots, A_{n}\right\}$ is weak group-envyfree if for any pair of coalitions $N_{1}, N_{2}$ with $\left|N_{1}\right|=\left|N_{2}\right|$ there is no division $\left\{C_{i}\right\}_{i \in N_{1}}$ of $\cup_{j \in N_{2}} A_{j}$ such that $C_{i} \in P_{i}\left(A_{i}\right)$ for all $i \in N_{1}$.

This definition is adapted from Berliant et al. (1992). Obviously, if an allocation is weak-group envy-free then it is envy-free and weakly Pareto efficient.

Definition 4.2'. A division $\left\{A_{1}, A_{2}, \ldots, A_{n}\right\}$ is group-envy-free if for any pair of coalitions $N_{1}, N_{2}$ with $\left|N_{1}\right|=\left|N_{2}\right|$ there is no division $\left\{C_{i}\right\}_{i \in N_{1}}$ of $\cup_{j \in N_{2}} A_{j}$ such that $A_{i} \notin P_{i}\left(C_{i}\right)$ for all $i \in N_{1}$ and $C_{i} \in P_{i}\left(A_{i}\right)$ at least for one $i \in N_{1}$.

Of course when preferences $P_{i}$ are derived from rational weak preferences $\succcurlyeq_{i}$, (equivalently, $\succ_{i}$ are negative transitive and asymmetric), then the last part of Definition $4.2^{\prime}$ reads as

$C_{i} \succcurlyeq_{i} A_{i}$ for all $i \in N_{1}$ and $C_{i} \succ_{i} A_{i}$ at least for one $i \in N_{1}$.

As in Proof of Proposition 3.3 it can be shown that under the assumptions of this proposition every weak group-envy-free division is group-envy-free, that is, the two concepts coincide.

Theorem 4.4. Under the assumptions of Theorem 3.1 there exists a weak group-envy-free and weakly Pareto efficient allocation.

Proof of this theorem will be given in Appendix B. 
Let $\mu_{i}^{j}, i \in N$ be nonatomic measures on $(X, \Sigma)$. If there had existed a partition of $X$ into $n$ parts, say $C_{j}, j \in N$, so that restrictions of vector-measure $\mu=\left(\mu_{1}, \mu_{2}, \ldots, \mu_{n}\right)$ into sets $C_{j}, j \in N$ have identical ranges, then one could exploit the standard scheme of a proof of the existence of a fair division by assigning each individual $j$ the piece $C_{j}$. The author does not know whether such a partition exists, therefore we are not able to derive the existence of a fair division from the existence of an equilibrium division.

For proving the existence of a fair (or more generally, a groupenvy-free and efficient) allocation, we will use the aforementioned method that we also use to establish the existence of a competitive equilibrium: We will first construct an economy with an aggregate endowment set, and then generate from it the type of economy as in Gale and Mas-Colell (1975), in which individuals are given equal profits. We will then use a competitive equilibrium of the latter economy for constructing a division in the measurable space division problem that is group-envy-free and Pareto efficient.

When $X$ is a subset of the Euclidean space $R^{k}$ and preferences $\succ_{i}$ are given by scalar Borel measures on $X$, absolutely continuous with respect to the Lebesgue measure, Theorem 4.1 reduces to Theorem 2 of Berliant et al. (1992). It should be noted that in their approach they start with a special reference measure (the Lebesgue measure), while our approach does not assume any such a priori measure.

Theorem 4.1 implies the following corollary:

Corollary 4.5. Under the assumptions of Theorem 3.1 there exists a fair division of a measurable space $(X, \Sigma)$.

Finally, let us also note that if each agent $i$ has a single attribute formalized as a finite positive measure $\mu_{i}$ on $\Sigma$ and preferences defined simply as strictly greater relation on $R$ (which is the same as saying that preferences are given by scalar measures on $\Sigma$ ), then Corollary 4.2 is reduced to Weller's (1985) fairness result.

\section{Appendix A. Chernoff's theorem}

The following theorem is a generalization of a result known as Dubins-Spanier's theorem (see also Aliprantis and Border, 1994, p. 358) and easily follows from this result. It is to be noted that, in fact, this theorem was discovered a decade earlier by Chernoff (1951). Both of these theorems are consequences of the celebrated Liapunov Theorem (Liapunov, 1940).

Theorem A. Let $(X, \Sigma)$ be a measurable space and let $\mu_{1}, \mu_{2}, \ldots$, $\mu_{n}$ be nonatomic finite vector measures on $(X, \Sigma)$ of dimensions $s_{1}, s_{2}$ $\ldots, s_{n}$, respectively. Then the following set in $R^{s}$, where $s=\sum_{j=1}^{n} s_{j}$,

$\mathcal{R}=\left\{\left(\mu_{i}\left(B_{i}\right)\right)_{i=1}^{n} \in R^{s} \mid P=\left(B_{1}, B_{2}, \ldots, B_{n}\right)\right.$ is a division of $\left.X\right\}$

is compact and convex.

Proof of Theorem A is based on the Dubins-Spanier's theorem. Let $\mu=\left(\mu_{k}\right)_{k=1}^{s}$ be a vector measure $\left(\mu_{1}, \mu_{2}, \ldots, \mu_{n}\right)$ of dimension $s$. With every division $P=\left(B_{1}, B_{2}, \ldots, B_{n}\right) \in \Pi^{n}(X)$ we associate the $s \times n$ matrix of reals $M(P)=\left(\mu_{k}\left(B_{i}\right)\right)$. Denote by $M^{s \times n}$ the space of all $s \times n$ matrices with real entries. By Theorem 1 in Dubins and Spanier (1961) the range $\mathcal{R}^{\prime} \subset M^{s \times n}$ of the matrix-valued function $M$ is compact and convex.

Let $L: M^{S \times n} \rightarrow R^{S}$ be a mapping defined in the following way: The first $s_{1}$ components of $L(M)$ are the first $s_{1}$ entries in the first column of matrix $M$, the second $s_{2}$ components are the entries in the second column of $M$ with the column indexes $s_{1}+1$ through $s_{1}+s_{2}$, and so on. Clearly, $L$ is a linear mapping with $L\left(\mathcal{R}^{\prime}\right)=\mathcal{R}$. Since $\mathcal{R}^{\prime}$ is compact and convex it follows that so is $\mathcal{R}$.

\section{Appendix B. Proofs of main results}

Proof of Theorem 3.1. We will reduce the above exchange economy to an economy of exchange of a finite number of divisible homogeneous commodities, where endowments of individuals are sets in the consumption spaces, rather than commodity bundles, from which the individuals are free to choose.

There are $s$ commodities in this economy. Thus the commodity space is $R^{S}$, the $s$-dimensional Euclidean space. $R_{+}^{S}$ and $R_{++}^{s}$ denote the nonnegative and positive cones in this space, respectively. For $i \in N, R_{+}^{s_{i}}$ will be the consumption space of individual $i$, which we consider as a subset in $R_{+}^{s}$.

We define the initial endowment set $E_{i} \subset R^{S}$ of individual $i$ in the following way:

$E_{i}=\left\{\left(\mu_{1}\left(C_{1}\right), \mu_{2}\left(C_{2}\right), \ldots, \mu_{n}\left(C_{n}\right)\right) \mid\left\{C_{1}, C_{2}, \ldots, C_{n}\right\}\right.$ is a partition of $\left.A_{i}\right\}$.

By Theorem A the initial endowment sets are compact and convex.

Denote by $\Delta$ the unit simplex in $R^{s}$. A price $p=\left(p^{s_{1}}, \ldots, p^{s_{n}}\right)$, where $p^{s_{i}}$ is the price associated with consumer $i$, will be an element of $\Delta$. Given a price $p \in \Delta$, the wealth of individual $i$ is defined as

$\alpha_{i}(p)=\max \left\{p \cdot x \mid x \in E_{i}\right\}$ for all $i \in N$,

where $p \cdot x$ is the scalar product of vectors $p$ and $x$. The budget set of $i$ is defined as

$\mathcal{B}_{i}(p)=\left\{x \in R^{s_{i}} \mid p^{s_{i}} \cdot x \leq \alpha_{i}(p)\right\}$

Preferences of individual $i$ are defined through mapping $P_{i}$ : $R_{+}^{S_{i}} \rightarrow R_{+}^{s_{i}}$ which is irreflexive, that is, $x_{i} \notin P_{i}\left(x_{i}\right)$ for all $x_{i} \in R_{+}^{s_{i}}$, has an open graph in $R_{+}^{S_{i}} \times R_{+}^{S_{i}}$ and nonempty convex values.

We denote by $\mathcal{E}$ the exchange economy involving $n$ individuals $1, \ldots, n$, their endowment sets $E_{1}, \ldots, E_{n}$, and preferences $P_{1} \ldots$, $P_{n}$, respectively.

Define the set of aggregate endowment vectors as the algebraic sum of individual endowment sets, $E=\sum_{i \in N} E_{i}$, and the technology set as $Y=E+R_{-}^{S}$.

Proof of the following fact is a straightforward exercise.

Fact 1. $Y$ is closed and has a nonempty bounded intersection with the nonnegative cone $R_{+}^{s}$.

Let $E_{0} \subset E$ be the Pareto frontier of $Y$, in other words, the smallest set with $Y=E_{0}+R_{-}^{s}$.

Definition B1. A competitive equilibrium in economy $\mathcal{E}$ is defined as an $(2 N+1)$-tuple $\left(\bar{x}_{1}, \bar{x}_{2}, \ldots, \bar{x}_{n}, \hat{y}_{1}, \hat{y}_{2}, \ldots, \hat{y}_{n}, \bar{p}\right) \in\left(\left(\Pi_{i \in N} R_{+}^{s_{i}}\right) \times\right.$ $\left.\left(\prod_{i \in N} E_{i}\right)\right) \times \Delta$ such that

$\bar{x}_{0}=\sum_{i \in N} \bar{x}_{i}=\sum_{i \in N} \hat{y}_{i}=\hat{y}_{0} \in E_{0}$,

$\bar{p} \cdot \bar{x}_{i}=\bar{p} \cdot \hat{y}_{i}=\alpha_{i}(\bar{p})$ for $\quad i \in N$,

and

$P\left(\bar{x}_{i}\right) \cap \mathcal{B}_{i}(\bar{p})=\varnothing \quad$ for $\quad i \in N$.

Define

$\Pi(p)=\sup p \cdot Y$ for $p \in \Delta$

Obviously the supremum in formula (4) is attained for each $p \in \Delta$.

Proof of the following fact is a straightforward exercise.

Fact 2. $\Pi: \Delta \rightarrow R_{+}$is a nonnegative continuous function. 
By the definitions of $Y$ and $E$ we have

$\Pi(p)=\sum_{i \in N} \alpha_{i}(p)$ for $p \in \Delta$.

(This is known as 'aggregation' in Microeconomics; see Mas-Colell et al., 1995, Proposition 5.E.1). Following Gale and Mas-Colell (1975) observe that

$\bar{p} \cdot \hat{y}_{0}=\sum_{i \in N} \bar{p} \cdot \hat{y}_{i}=\sum_{i \in N} \alpha_{i}(\bar{p})=\max \bar{p} \cdot Y$.

By the positive endowments assumption, $E_{i}$ contains a strictly positive vector. Hence

$\alpha_{i}(p)>0 \quad$ for all $p \in \Delta, \quad i \in N$.

Now we have the following economy

$\mathcal{E}_{0}=\left\{\left(R^{S_{i}}, P_{i}, \alpha_{i}\right)_{i \in N}, Y\right\}$

satisfying all of the assumptions of Gale-Mas-Colell existence theorem (1975). So there exists an $(N+1)-$ tuple $\left(\bar{x}_{1}, \bar{x}_{2}, \ldots, \bar{x}_{n}, \bar{p}\right)$ such that the following relations are satisfied:

$\sum_{i \in N} \bar{x}_{i}=\bar{x}_{0} \in Y$,

$\bar{p} \cdot \bar{x}_{i}=\alpha_{i}(\bar{p})$ for $i \in N$,

and

$P\left(\bar{x}_{i}\right) \cap \mathcal{B}_{i}(\bar{p})=\varnothing \quad$ for $\quad i \in N$.

Next we will show that for every competitive equilibrium $\left(\bar{x}_{1}, \bar{x}_{2}, \ldots, \bar{x}_{n}, \bar{p}\right)$ in $\mathcal{E}_{0}$ (that is for every vector $\left(\bar{x}_{1}, \bar{x}_{2}, \ldots, \bar{x}_{n}, \bar{p}\right)$ satisfying conditions (6)-(8)), there exist $\hat{y}_{i}, i \in N$ such that $\left(\bar{x}_{1}, \bar{x}_{2}, \ldots, \bar{x}_{n}, \hat{y}_{1}, \hat{y}_{2}, \ldots, \hat{y}_{n}, \bar{p}\right)$ is a competitive equilibrium in $\mathcal{E}$.

In this step we make use of the monotonicity assumption. So let $\left(\bar{x}_{1}, \bar{x}_{2}, \ldots, \bar{x}_{n}, \bar{p}\right)$ be a competitive equilibrium in $\mathcal{E}_{0}$. Then $\sum_{i \in N} \bar{x}_{i}=\bar{x}_{0} \in Y$.

If $\bar{x}_{0} \in E_{0}$, then since $E_{0} \subset E$

$\bar{x}_{0}=\sum_{i \in N} \bar{x}_{i}=\sum_{i \in N} \hat{y}_{i}$

for some $\hat{y}_{i} \in E_{i}(i \in N)$. Since $\bar{p} \cdot \bar{x}_{0}=\max \bar{p} \cdot Y$ it follows that Eq. (2) are satisfied. Thus $\left(\bar{x}_{1}, \bar{x}_{2}, \ldots, \bar{x}_{n}, \hat{y}_{1}, \hat{y}_{2}, \ldots, \hat{y}_{n}, \bar{p}\right)$ is a competitive equilibrium in $\mathcal{E}$. Assume $\bar{x}_{0} \notin E_{0}$. It follows from the definitions of $Y$ and $E_{0}$ that there exists $\hat{x}_{0} \in E_{0}$ such that $\hat{x}_{0} \geq \bar{x}_{0}$. Set $\hat{x}_{i}=\bar{x}_{i}+$ $\left(\hat{x}_{0}-\bar{x}_{0}\right)^{s_{i}}$ for $i \in N$. Then

$\sum_{i \in N} \hat{x}_{i}=\sum_{i \in N} \bar{x}_{i}+\sum_{i \in N}\left(\hat{x}_{0}-\bar{x}_{0}\right)^{s_{i}}=\hat{x}_{0}$.

So $\left(\hat{x}_{1}, \hat{x}_{2}, \ldots, \hat{x}_{n}\right)$ is feasible.

We also have

$\bar{p} \cdot \hat{x}_{i}=\bar{p} \cdot \bar{x}_{i}=\alpha_{i}(\bar{p})$ for $\quad i \in N$.

Indeed, $\hat{x}_{i} \geq \bar{x}_{i}$ implies $\bar{p} \cdot \hat{x}_{i} \geq \bar{p} \cdot \bar{x}_{i}=\alpha_{i}(\bar{p})$ for all $i$. It is not possible that $\bar{p} \cdot \hat{x}_{j}>\bar{p} \cdot \hat{x}_{j}$ for some $j$; for otherwise, we would have

$\bar{p} \cdot \sum_{i \in N} \hat{x}_{i}=\sum_{i \in N} \bar{p} \cdot \hat{x}_{i}>\sum_{i \in N} \bar{p} \cdot \bar{x}_{i}=\sum_{i \in N} \alpha_{i}(\bar{p})=\max \bar{p} \cdot Y$.

This is a contradiction since vectors $\hat{x}_{i}$ form a feasible allocation. Therefore $\bar{p} \cdot \hat{x}_{i} \leq \bar{p} \cdot \bar{x}_{i}$ and hence $\bar{p} \cdot \hat{x}_{i}=\bar{p} \cdot \bar{x}_{i}$ for all $i$.

Since $\hat{x}_{i} \geq \bar{x}_{i}$, by the monotonicity assumption it follows that $P_{i}\left(\hat{x}_{i}\right) \subset P_{i}\left(\bar{x}_{i}\right)$. This inclusion, together with Eq. (8) imply that

$P_{i}\left(\hat{x}_{i}\right) \cap B_{i}(\bar{p})=\varnothing$ for $i \in N$.
So, we have constructed a new competitive equilibrium $\left(\hat{x}_{1}, \hat{x}_{2}, \ldots, \hat{x}_{n}, \bar{p}\right)$ in $\mathcal{E}_{0}$ such that

$\sum_{i \in N} \hat{x}_{i}=\hat{x}_{0} \in E_{0}$.

We have shown above how to construct a competitive equilibrium in $\mathcal{E}$ from one in $\mathcal{E}_{0}$ with this property. Thus, we have proven the existence of a competitive equilibrium in economy $\mathcal{E}$.

Let $\left(\bar{x}_{1}, \bar{x}_{2}, \ldots, \bar{x}_{n}, \hat{y}_{1}, \hat{y}_{2}, \ldots, \hat{y}_{n}, \bar{p}\right)$ be a competitive equilibrium in the economy $\mathcal{E}$. By the definition of sets $E_{i}(i \in N)$ there are divisions $P_{i}=\left\{A_{i}^{1}, A_{i}^{2}, \ldots, A_{i}^{n}\right\}$ of sets $A_{i}$ such that $\left(\mu_{1}\left(A_{i}^{1}\right), \mu_{2}\left(A_{i}^{2}\right), \ldots, \mu_{n}\left(A_{i}^{n}\right)\right)=\hat{y}_{i}$ for each $i \in N$. Set $B_{j}=\cup_{i \in N} A_{i}^{j}(j \in N)$. Clearly, $\left\{B_{1}, B_{2}, \ldots, B_{n}\right\}$ is a division of $X$. Define a measure $v$ on $\Sigma$ by setting

$v(D)=\sum_{i \in N} \bar{p}^{s_{i}} \cdot \mu_{i}\left(D \cap B_{i}\right)$ for $D \in \Sigma$.

Obviously $v$ is a measure on $\Sigma$ that is absolutely continuous with respect to $\theta=\sum_{i \in N} \sum_{j=1}^{s_{i}} \mu_{i}^{j}$. We will show that the pair $\left(\left\{B_{1}, B_{2}, \ldots, B_{n}\right\}, v\right)$ is a competitive equilibrium in the measurable space exchange economy. As $\left\{B_{1}, \ldots, B_{n}\right\}$ is a division of $X$, it suffices to show that $B_{i}$ is $>{ }_{i}$-maximal in the budget set of individual $i, \mathcal{B}_{i}(v)$, for all $i \in N$. Assume on the contrary, that for some $i$ there exists $B \in \mathcal{B}_{i}(v)$ such that $B \succ_{i} B_{i}$. Thus

$v(B) \leq v\left(A_{i}\right)=v\left(B_{i}\right)=\alpha_{i}(\bar{p})$

and $\mu_{i}(B) \in P_{i}\left(\mu_{i}\left(B_{i}\right)\right)$. This preference implies that

$\bar{p}^{s_{i}} \cdot \mu_{i}(B)>\bar{p}^{s_{i}} \cdot \mu_{i}\left(B_{i}\right)=v\left(B_{i}\right)=\alpha_{i}(\bar{p}) \geq v(B)=\sum_{j \in N} \bar{p}^{s_{j}} \cdot \mu_{j}\left(B \cap B_{j}\right)$.

We thus have

$\bar{p}^{s_{i}} \cdot \mu_{i}\left(B \backslash B_{i}\right)>\sum_{j \in N \backslash\{i\}} \bar{p}^{s_{j}} \cdot \mu_{j}\left(B \cap B_{j}\right)$.

As $\left\{B_{1}, \ldots, B_{n}\right\}$ is a division of $X, B \backslash B_{i}=\cup_{j \in N \backslash\{i\}}\left(B \cap B_{j}\right)$, where sets $B \cap B_{j}(j \in N \backslash\{i\})$ are disjoint. It follows from the last two relations that there exists $j \neq i$ such that

$\bar{p}^{s_{i}} \cdot \mu_{i}\left(B \cap B_{j}\right)>\bar{p}^{s_{j}} \cdot \mu_{j}\left(B \cap B_{j}\right)$.

This implies $\mu_{i}\left(B \cap B_{j}\right) \geq 0$. The last inequality implies that the owners of the piece $B \cap B_{j}$ would receive a higher profit by selling this piece to individual $i$ rather than to individual $j$. This contradicts to the construction of the division $\left\{B_{1}, \ldots, B_{n}\right\}$ as a profit maximizing division.

Remark B. A byproduct of the above proof is the existence of a competitive equilibrium in an exchange economy where individuals are free to choose from some sets of commodity bundles rather than possessing a single commodity bundle. Such economies were considered in Aubin (1981).

\section{Proof of Theorem 4.1. Define}

$E=\left\{\left(\mu_{1}\left(A_{1}\right), \mu_{2}\left(A_{2}\right), \ldots, \mu_{n}\left(A_{n}\right)\right) \mid\left\{A_{1}, A_{2}, \ldots, A_{n}\right\}\right.$ is a partition of $\left.X\right\}$.

By Theorem A, $E \subset R^{s}$ is a nonempty compact convex set. Set $Y=E+R_{-}^{S}$ and $X_{i}=R_{+}^{S_{i}}$ for $i \in N$ as in the previous proof. As before, define

$\alpha(p)=\max p \cdot Y$.

Define individual wealth functions by setting $\alpha_{i}(p)=\alpha(p) / n$ for $i \in N$. It is easily seen that $\alpha(p)>0$ and hence

$\alpha_{i}(p)>0$ for all $p \in \Delta$. 
Budget sets are defined as

$B_{i}(p)=\left\{x_{i} \in X_{i} \mid p^{s_{i}} \cdot x_{i} \leq \alpha_{i}(p)\right\}$

So, we have an economy $\mathcal{E}$ for which a competitive equilibrium is defined in the following way:

Definition B2. An $(n+1)$-tuple $\left(\bar{x}_{1}, \bar{x}_{2}, \ldots, \bar{x}_{n}, \bar{p}\right)$, where $\bar{x}_{i} \in X_{i} \bar{x}_{0}=\Sigma_{i \in N} \bar{x}_{i} \in Y, \bar{p} \cdot \bar{x}_{0}=\max \bar{p} \cdot Y$, and $\bar{p} \in \Delta$ is a competitive equilibrium in economy $\mathcal{E}$, if $\bar{x}_{i}$ is a $P_{i}$-maximal element in the budget set $B_{i}(p)$ for all $i \in N$.

For the economy $\mathcal{E}$ all of the conditions of Gale-Mas-Colell existence theorem (1975) are satisfied. So, there exists a competitive equilibrium $\left(\bar{x}_{1}, \bar{x}_{2}, \ldots, \bar{x}_{n}, \bar{p}\right)$ in economy $\mathcal{E}$. We have

$\bar{x}_{0}=\sum_{i \in N} \bar{x}_{i} \in Y \quad$ and $\quad \bar{p} \cdot \bar{x}_{0}=\max \bar{p} \cdot Y$.

If $\bar{x}_{0} \in E_{0}$, where, as before, $E_{0}$ is the Pareto frontier of $Y$, then $\bar{x}_{0} \in E$, and hence there exists a division $\left\{B_{1}, B_{2}, \ldots, B_{n}\right\}$ of $X$ such that

$\mu_{i}\left(B_{i}\right)=\bar{x}_{i}$ for $i \in N$.

As in the previous proof, define a measure $v$ on $\Sigma$ by setting

$v(D)=\sum_{i \in N} \bar{p}^{s_{i}} \cdot \mu_{i}\left(D \cap B_{i}\right)$ for $D \in \Sigma$.

We have

$\nu\left(B_{j}\right)=\bar{p}^{s_{j}} \cdot \mu_{j}\left(B_{j}\right)=\alpha_{j}(p)=\frac{\alpha(\bar{p})}{n}$ for all $j \in N$.

We assert that division $B=\left\{B_{1}, B_{2}, \ldots, B_{n}\right\}$ is weak groupenvy-free and weakly Pareto efficient. Assume it is not weak group-envy-free. Then there exist $N_{1}, N_{2} \subset N$ such that $\left|N_{1}\right|=\left|N_{2}\right|$ and there is a division $\cup_{i \in N_{1}} C_{i}$ of $\cup_{j \in N_{2}} B_{j}$ such that $C_{i} \in P_{i}\left(B_{i}\right)$ for all $i \in N_{1}$. It follows then $v\left(C_{i}\right)>v\left(B_{i}\right)$ for all $i \in N_{1}$. Summing up these inequalities we will have $v\left(\cup_{i \in N_{1}} C_{i}\right)>v\left(\cup_{i \in N_{1}} B_{i}\right)$. But from (9) we have

$\nu\left(\cup_{i \in N_{1}} C_{i}\right)=v\left(\cup_{j \in N_{2}} B_{j}\right)=\frac{\left|N_{2}\right|}{n} \alpha(\bar{p})=\nu\left(\cup_{i \in N_{1}} B_{i}\right)$.
Assume now that division $B$ is not weakly Pareto efficient. Then there exists a division $C=\left\{C_{1}, C_{2}, \ldots, C_{n}\right\}$ of $X$ such that $C_{i} \in P_{i}\left(B_{i}\right)$ for all $i \in N$. Then $v\left(C_{i}\right)>\mu_{i}\left(B_{i}\right)$ for all $i \in N$. Summing these inequalities we will have $\sum_{i \in N} v\left(C_{i}\right)>\sum_{i \in N} v\left(B_{i}\right)$ that is, $v(X)>v(X)$, a contradiction.

If $\bar{x}_{0} \notin E_{0}$, then there exists $\hat{x}_{0} \geq \bar{x}_{0}$ such that $\hat{x}_{0} \in E_{0}$, and hence $\hat{x}_{0} \in E$. Using the weak monotonicity assumption as in Proof of Theorem 3.1 we reduce the situation to the case of $\bar{x}_{0} \in E_{0}$.

\section{References}

Aliprantis, C., Border, K.C., 1994. Infinite Dimensional Analysis. Springer Verlag, Berlin.

Aubin, J.-P., 1981. Cooperative fuzzy games. Math. Oper. Res. 6, 1-13.

Bewley, T., 1972. Existence of equilibria in economies with infinitely many commodities. J. Econ. Theory 4, 514-540.

Berliant, M., 1985. An equilibrium existence result for an economy with land. J. Math. Econ. 14, 53-56.

Berliant, M., Dunz, K., (1986). The welfare theorems and economics with land and a finite number of traders. Working paper, No 54, Rochester Center for Economic Research.

Berliant, M., Thomson, W., Dunz, K., 1992. On the fair division of a heterogeneous commodity. J. Math. Econ. 21, 201-216.

Berliant, M., Dunz, K., 2004. A foundation of location theory: existence of equilibrium, the welfare theorems, and the core. J. Math. Econ. 40, 593618.

Chambers, C.P., 2005. Allocation rules for land division. J. Econ. Theory 121, 236258.

Chernoff, H., 1951. An extension of a result of Liapunoff on the range of a vector measure. Proc. Am. Math. Soc. 2, 722-726.

Dubins, L.E., Spanier, E.H., 1961. How to cut a cake fairly. Am. Math. Monthly 68, $1-17$.

Dunz, K., 1991. On the core of a land trading game. Reg. Sci. Urban Econ. 21, 7388.

Gale, D., Mas-Colell, A., 1975. An equilibrium existence theorem for a general model without ordered preferences. J. Math. Econ. 2, 9-16.

Hüsseinov, F., 2008. Existence of the core in a heterogeneous divisible commodity exchange economy. Int. J. Game Theory 37, 387-395.

Liapunov, A.A., 1940. Sur les fonctions-vecteurs completement additives. Bull. Acad. Sci. USSR 4, 465-478.

Mas-Colell, A., Whinston, M.D., Green, J.R., 1995. Microeconomic Theory. Oxford University Press, New-York/Oxford.

Weller, D., 1985. Fair division of a measurable space. J. Math. Econ. 14, 5-17. 